Delft University of Technology

\title{
Calibrated quantum thermometry in cavity optomechanics
}

Chowdhury, A.; Vezio, P.; Bonaldi, M.; Borrielli, A.; Marino, F.; Morana, B.; Pandraud, G.; Sarro, P. M.; Serra, E.; More Authors

DOI

10.1088/2058-9565/ab05f1

Publication date

2019

Document Version

Final published version

Published in

Quantum Science and Technology

\section{Citation (APA)}

Chowdhury, A., Vezio, P., Bonaldi, M., Borrielli, A., Marino, F., Morana, B., Pandraud, G., Sarro, P. M., Serra, E., \& More Authors (2019). Calibrated quantum thermometry in cavity optomechanics. Quantum Science and Technology, 4(2), 1-9. [024007]. https://doi.org/10.1088/2058-9565/ab05f1

\section{Important note}

To cite this publication, please use the final published version (if applicable).

Please check the document version above.

\section{Copyright}

Other than for strictly personal use, it is not permitted to download, forward or distribute the text or part of it, without the consent of the author(s) and/or copyright holder(s), unless the work is under an open content license such as Creative Commons.

Takedown policy

Please contact us and provide details if you believe this document breaches copyrights.

We will remove access to the work immediately and investigate your claim. 
Green Open Access added to TU Delft Institutional Repository

'You share, we take care!' - Taverne project

https://www.openaccess.nl/en/you-share-we-take-care

Otherwise as indicated in the copyright section: the publisher is the copyright holder of this work and the author uses the Dutch legislation to make this work public. 


\section{PAPER}

\section{Calibrated quantum thermometry in cavity optomechanics}

To cite this article: A Chowdhury et al 2019 Quantum Sci. Technol. 4024007

View the article online for updates and enhancements.

\section{Recent citations}

$$
\begin{aligned}
& \text { - Active feedback cooling of a SiN } \\
& \frac{\text { membrane resonator by electrostatic }}{\text { actuation }} \\
& \text { A. Borrielli et al } \\
& \text { - Mechanical oscillator thermometry in the } \\
& \frac{\text { nonlinear optomechanical regime }}{\text { V. Montenegro et al }} \\
& \text { - Quantum motion of a squeezed } \\
& \frac{\text { mechanical oscillator attained via an }}{\text { optomechanical experiment }} \\
& \text { P. Vezio et al }
\end{aligned}
$$

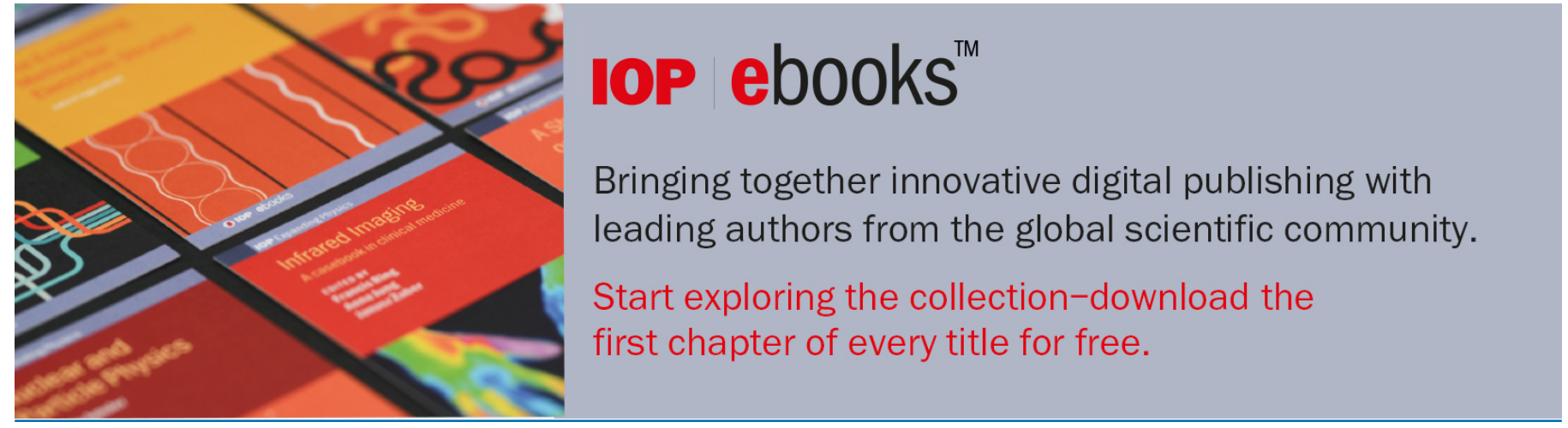

This content was downloaded from IP address 131.180 .231 .191 on $03 / 08 / 2021$ at 10:25 


\section{Quantum Science and Technology}

\section{PAPER}

\section{Calibrated quantum thermometry in cavity optomechanics}

RECEIVED

22 November 2018

REVISED

21 January 2019

ACCEPTED FOR PUBLICATION

11 February 2019

PUBLISHED

5 March 2019

\author{
A Chowdhury ${ }^{1,2} \oplus$, P Vezio $^{2,3}$, M Bonaldi $^{4,5}$, A Borrielli $^{4,5}$, F Marino $^{1,2}$, B Morana $^{4,6}$, G Pandraud $^{6}$, \\ A Pontin ${ }^{7}$ (i), G A Prodi ${ }^{5,8}$, P M Sarro $^{6}$, E Serra $^{5,6}$ and F Marin ${ }^{1,2,3,9}$ (i) \\ 1 CNR-INO, L.go Enrico Fermi 6, I-50125 Firenze, Italy \\ 2 Istituto Nazionale di Fisica Nucleare (INFN), Sezione di Firenze, Via Sansone 1, I-50019 Sesto Fiorentino (FI), Italy \\ 3 European Laboratory for Non-Linear Spectroscopy (LENS), Via Carrara 1, I-50019 Sesto Fiorentino (FI), Italy \\ 4 Institute of Materials for Electronics and Magnetism, Nanoscience-Trento-FBK Division, I-38123 Povo, Trento, Italy \\ 5 Istituto Nazionale di Fisica Nucleare (INFN), Trento Institute for Fundamental Physics and Application, I-38123 Povo, Trento, Italy \\ 6 Dept. of Microelectronics and Computer Engineering/ECTM-EKL, Delft University of Technology, Feldmanweg 17, 2628 CT Delft, The \\ Netherlands \\ 7 Department of Physics and Astronomy, University College London, Gower Street, London WC1E 6BT, United Kingdom \\ 8 Dipartimento di Fisica, Università di Trento, I-38123 Povo, Trento, Italy \\ 9 Dipartimento di Fisica e Astronomia, Università di Firenze, Via Sansone 1, I-50019 Sesto Fiorentino (FI), Italy \\ E-mail: marin@fi.infn.it
}

Keywords: optomechanics, quantum thermometry, micro-oscillators

\begin{abstract}
Cavity optomechanics has achieved the major breakthrough of the preparation and observation of macroscopic mechanical oscillators in non-classical states. The development of reliable indicators of the oscillator properties in these conditions is important also for applications to quantum technologies. We compare two procedures to infer the oscillator occupation number, minimizing the necessity of system calibrations. The former starts from homodyne spectra, the latter is based on the measurement of the motional sideband asymmetry in heterodyne spectra. Moreover, we describe and discuss a method to control the cavity detuning, that is a crucial parameter for the accuracy of the latter, intrinsically superior procedure.
\end{abstract}

\section{Introduction}

A crucial outcome of cavity optomechanics [1] is the observation of genuine quantum features in the behavior of macroscopic mechanical oscillators. The most relevant indicator of the achieved mechanical quantum domain is the so-called motional sideband asymmetry. The optomechanical interaction generates spectral peaks around the carrier frequency of a probe field, at distances equal to the mechanical oscillation frequency $\Omega_{\mathrm{m}}$. Their amplitudes are generally different according to quantum theory. Different interpretations have been proposed to explain such asymmetry [2-4], all agreeing in recognizing it as a non-classical signature of the mechanical oscillator [4], as soon as spurious experimental features are avoided [5, 6]. A particularly intuitive explanation considered that the anti-Stokes (blue) sideband implies an energy transfer from the oscillator to the radiation (frequency up-conversion of photons), and vice versa for the Stokes (red) sideband. Since the quantum oscillator cannot yield energy when it is in the ground state, the anti-Stokes process is less favored. It turns out that the blue and red sideband strengths are proportional respectively to $\bar{n}$ and $(\bar{n}+1)$ [7], where $\bar{n}$ is the mean occupation number of the oscillator.

Measurements of the sideband asymmetry have been extensively used to monitor the motion of trapped ions [8], and it has recently become a key technique for cavity optomechanics. Besides its utility as direct indicator of the oscillator quantum behavior, the sideband asymmetry is a powerful index to deduce the oscillator occupation number avoiding delicate evaluations of optomechanical parameters, such as oscillator effective mass or optomechanical gain, and calibrations of the detection system. It has been remarked that the thermal occupation number $\bar{n}_{\text {th }}$ allows a direct evaluation of the absolute temperature, and it is therefore of extraordinary potential metrological interest. Several experiments concerning the use of optomechanical quantum effects for the measurement of absolute temperature, covering the full range from ultra-cryogenic to 
room temperatures, are indeed been running $[9,10]$. As a matter of fact, we expect that accurate measurements of the oscillator displacement variance and of motional sideband asymmetry will be extensively exploited in the next future, and deserve a detailed investigation.

Sideband asymmetry has been measured in optical experiments by alternatively positioning a probe field at a detuning of $\pm \Omega_{\mathrm{m}}$ around the cavity resonance [11], as well as, in a single measurement, from the spectral sidebands in a probe field [12-15]. The former technique is particularly useful in the regime of deeply resolved sidebands $\left(\Omega_{\mathrm{m}} \gg \kappa\right.$, where $\kappa$ is the cavity linewidth), since for each position of the probe the measured sideband is at the cavity resonance frequency and it is thus amplified. On the other hand, the control of systematic effects can be an issue: the system should remain stable between two separate measurement sessions, the probe intensity and the detection efficiency must be equal for the two values of detuning, and the probe detuning itself must be very accurate. The latter technique, while introduced more recently in cavity optomechanics, is already well established, but it also requires an accurate control of the probe detuning, above all in the case of narrow cavity resonance. The cavity works indeed as frequency filter for the output radiation, with an effect that differs between the two sidebands and can thus spoil the measurement of their ratio.

In this work we experimentally investigate the sideband asymmetry as signature of quantum performance, and we compare it with a further indicator, i.e. the oscillator displacement variance measured form the area of the corresponding peak in the probe phase spectrum. Furthermore, we demonstrate a method for correcting the measured sideband asymmetry for non-null probe detuning, exploiting the spectral features of the device oscillating modes that are weakly coupled to the cavity radiation ('heavy' modes).

\section{Theoretical background}

The displacement spectrum of a mechanical oscillator is characterized by resonance peaks corresponding to the different normal modes. The area underlying each peak is a measure of the variance of the motion of the harmonic oscillator associated to the readout of that normal mode. It can be written as $\mathcal{A}_{x}=2 x_{\mathrm{ZPF}}^{2}(1 / 2+\bar{n})$ where $x_{\mathrm{ZPF}}=\sqrt{\hbar / 2 m_{\mathrm{eff}} \Omega_{\mathrm{m}}}$ is the zero-point fluctuations amplitude and $m_{\mathrm{eff}}$ is the oscillator effective mass. If the oscillator is in thermal equilibrium with a background at temperature $T_{\text {bath }}$, the mean thermal occupation number is $\bar{n}_{\text {th }} \simeq k_{\mathrm{B}} T_{\text {bath }} / \hbar \Omega_{\mathrm{m}}\left(k_{\mathrm{B}}\right.$ is the Boltzmann constant, and this expression of $\bar{n}_{\text {th }}$ is valid in the high temperature limit $\bar{n}_{\text {th }} \gg 1$ ), and the peak width is $\Gamma_{\mathrm{m}}=\Omega_{\mathrm{m}} / Q$, where $Q$ is the intrinsic mechanical quality factor.

When the mechanical oscillator is embedded in an optical cavity, the optomechanical interaction with intracavity radiation yields thermalization toward the photon bath at negligible occupation number ('backaction cooling' $[16,17])$, at a rate $\Gamma_{\text {opt }}$ proportional to the cooling laser power. The width of the spectral peak becomes $\Gamma_{\text {eff }}=\Gamma_{\mathrm{m}}+\Gamma_{\mathrm{opt}}$ and the oscillator occupation number is reduced by a factor of $\Gamma_{\text {eff }} / \Gamma_{\mathrm{m}}$. However, the back-action of the optomechanical measurement introduces an additional fluctuating force acting on the oscillator, that can be seen as the effect of the quantum noise in the radiation pressure. Since such quantum fluctuations are proportional to the laser power, and actually to $\Gamma_{\mathrm{opt}}$, the originated displacement noise of the optically damped oscillator has negligible dependence on the cooling power, in the limit $\Gamma_{\mathrm{opt}} \gg \Gamma_{\mathrm{m}}$. Its contribution to the total displacement variance can be written in terms of additional occupation number $\bar{n}_{\mathrm{BA}}^{\text {cool }}$ as $[1,18]$

$$
\bar{n}_{\mathrm{BA}}^{\text {cool }}=\left[\frac{\mathcal{L}\left(\Delta_{\text {cool }}+\Omega_{m}\right)}{\mathcal{L}\left(\Delta_{\text {cool }}-\Omega_{m}\right)}-1\right]^{-1},
$$

where $\mathcal{L}(\omega)=1 /\left[(\kappa / 2)^{2}+\omega^{2}\right]$ is the Lorentzian response function of the optical cavity with linewidth $\kappa$, and $\Delta_{\text {cool }}$ is the detuning of the cooling radiation with respect to the cavity resonance ( $\Delta>0$ if the radiation frequency is higher than the cavity resonant frequency).

The oscillator motion implies variations of the optical cavity resonance frequency $\omega_{\text {cav }}$, at the rate $G=-\partial \omega_{\text {cav }} / \partial x$. Such frequency fluctuations can be measured by exploiting the optical field leaving the cavity. The readout of the oscillator motion may be performed by analyzing the same radiation used for cooling. However, such radiation is commonly strongly detuned from the cavity resonance to assure an efficient cooling, therefore the optical susceptibility of the cavity is not trivial to be accurately considered [19]. It is often useful to introduce an additional, resonant probe field. The drawback is its additional back-action, that increases the oscillator noise. The probe back-action force does not depend on the cooling power, and it has the same effect of an increased background temperature. In general, the quantum radiation pressure noise produced by an intracavity field at detuning $\Delta$ is proportional to $\bar{n}_{\text {cav }}^{\max } \mathcal{L}(\Delta)\left[\mathcal{L}\left(\Delta+\Omega_{\mathrm{m}}\right)+\mathcal{L}\left(\Delta-\Omega_{\mathrm{m}}\right)\right]$ where $\bar{n}_{\text {cav }}^{\text {max }}$ is the average number of intracavity photons in case of resonant radiation, that is proportional to the input power. This expression allows us to write the oscillator occupation number added by the probe field in the form 


$$
\bar{n}_{\mathrm{BA}}^{\text {probe }}=\bar{n}_{\mathrm{BA}}^{\text {cool }} \frac{P^{\text {probe }}}{P^{\text {cool }}} \frac{\mathcal{L}\left(\Delta_{\text {probe }}\right)}{\mathcal{L}\left(\Delta_{\text {cool }}\right)} \frac{\mathcal{L}\left(\Delta_{\text {probe }}+\Omega_{\mathrm{m}}\right)+\mathcal{L}\left(\Delta_{\text {probe }}-\Omega_{\mathrm{m}}\right)}{\mathcal{L}\left(\Delta_{\text {cool }}+\Omega_{\mathrm{m}}\right)+\mathcal{L}\left(\Delta_{\text {cool }}-\Omega_{\mathrm{m}}\right)},
$$

where $P^{\text {probe/cool }}$ is the input power of the probe/cooling beam. Expressions (1) and (2) are particularly useful in the analysis of the experimental results, since they do not require the evaluation of the laser coupling efficiency and the consequent intracavity photon number, that are often not trivial. We just notice that, for an accurate evaluation of $\bar{n}_{\mathrm{BA}}^{\text {probe }}$, the probe and cooling beams should have the same level of mode-matching to the optical cavity. We remark that $\bar{n}_{\mathrm{BA}}^{\text {probe }}$ is proportional to $1 / P^{\mathrm{cool}}$ and actually to $1 / \Gamma_{\mathrm{opt}}$, provided that the probe field is close to resonance and has therefore a negligible effect on the effective width.

In conclusion, the total effective occupation number can be written as

$$
\bar{n}=\bar{n}_{\mathrm{th}} \frac{\Gamma_{\mathrm{m}}}{\Gamma_{\mathrm{eff}}}+\bar{n}_{\mathrm{BA}}^{\text {cool }}+\bar{n}_{\mathrm{BA}}^{\text {probe }} .
$$

A useful parameter to be considered is the area $\times$ width product $\mathcal{A} \Gamma_{\text {eff }}$ of the spectral peak, that we consider in the Lorentzian approximation, justified if $\Gamma_{\text {eff }} \gg \Omega_{\mathrm{m}}$. In the classical limit, when the variance of the motion is still dominated by thermal noise, such product should remain constant as the cooling power is increased, keeping, in the displacement spectrum, the value of $\mathcal{A}_{x} \times \Gamma_{\text {eff }} \simeq 2 x_{\mathrm{ZPF}}^{2} \bar{n}_{\mathrm{th}} \Gamma_{\mathrm{m}}=k_{\mathrm{B}} T_{\mathrm{bath}} / m_{\mathrm{eff}} \Omega_{\mathrm{m}} Q$. Quantum noise is instead at the origin of a linear increase of $\mathcal{A} \Gamma_{\text {eff }}$ versus $\Gamma_{\text {eff }}$. The peak area $\times$ width product in the frequency spectrum can be written as

$$
\mathcal{A} \Gamma_{\mathrm{eff}}=2 g_{0}^{2} \Gamma_{\mathrm{m}}\left[\bar{n}_{\mathrm{th}}+\left(\bar{n}_{\mathrm{BA}}^{\text {cool }}+\bar{n}_{\mathrm{BA}}^{\text {probe }}+\frac{1}{2}\right) \frac{\Gamma_{\mathrm{eff}}}{\Gamma_{\mathrm{m}}}\right],
$$

where the vacuum optomechanical coupling strength is $g_{0}=G x_{\mathrm{ZPF}}$. The accurate independent estimate of $g_{0}$ is not banal, since it crucially relies on the readout calibration and laser coupling efficiency [19, 20]. On the other hand, the terms into square brackets in equation (4), i.e. operatively, the ratio between the slope and the intercept in the $\mathcal{A} \Gamma_{\text {eff }}$ versus $\Gamma_{\text {eff }}$ line, is directly linked to the oscillator quantum state. It can be used as a check of the agreement between the expected and the measured behavior of the optomechanical system, i.e. to verify the absence of unmodeled extra noise, as well as, e.g. for evaluating $\bar{n}_{\text {th }}$ and actually the oscillator thermodynamic temperature $T_{\text {bath }}$.

When the occupation number is not too high, its more accurate measurement can be obtained from the heterodyne spectra of the radiation reflected by the cavity, that allow to distinguish the two sidebands produced by the Stokes and anti-Stokes processes in the optomechanical interaction. For a resonant probe, the sidebands peaks have areas proportional respectively to $\bar{n}$ (anti-Stokes) and $\bar{n}+1$ (Stokes), therefore $\bar{n}$ is directly calculated from the Stokes to anti-Stokes sidebands ratio $R$ as $\bar{n}=1 /(R-1)$. This indicator has at least two interesting properties: it does not require a calibration of the measured spectra in terms of, e.g. oscillator displacement or frequency fluctuations, and it is robust against effects of possible misleading extra-noise, e g. amplitude and phase noise of the source, heating the oscillator. It should be remark, however, that correlated phase and amplitude noise in the probe field can produce spurious sideband asymmetry even at high occupation numbers [5]. Moreover the signal-to-noise ratio can be an issue, more crucial than in the previously discussed method.

A crucial concern for the sidebands thermometry is the residual detuning of the probe with respect to the cavity resonance. The two motional sidebands are indeed filtered by the cavity according to $\mathcal{L}\left(\Delta_{\text {probe }} \pm \Omega_{\mathrm{m}}\right)$, and such filtering effect modifies $R$ as soon as $\Delta_{\text {probe }} \neq 0$, thus spoiling the validity of the measurement. The main original contribution of our work is a method to control and evaluate such residual probe detuning and the consequent correction to the measured $R$.

\section{Experimental setup}

The measurements are performed on a circular SiN membrane with a thickness of $100 \mathrm{~nm}$ and a diameter of $1.64 \mathrm{~mm}$, supported by a silicon ring frame. This frame is suspended on four points with alternating flexural and torsional springs, forming an on-chip 'loss shield' structure [21]. More information about the design, fabrication and the characteristic of the device can be found in Borrielli et al [22] and Serra et al [23, 24]. The theoretical resonance frequencies of the drum modes in a circular membrane are given by the expression $f_{m n}=f_{0} \alpha_{m n}$ where $\alpha_{m n}$ is the $n$th root of the Bessel polynomial $J_{m}$ of order $m$, and $f_{0}=\frac{1}{\pi} \sqrt{\frac{T}{\rho}} \frac{1}{\Phi}$ (Tis the stress, $\rho$ the density, $\Phi$ the diameter of the membrane). The measured frequencies are in close agreement (to better than $0.1 \%$ ) with the theoretical expression, where at cryogenic temperature $f_{0}=96.6 \mathrm{kHz}$. For $m>0$ we expect couples of degenerate modes. In the real device the perfect circular symmetry is broken, two orthogonal axes are defined and the two quasi-degenerate modes (the measured frequency split is below $100 \mathrm{~Hz}$ ) have shapes 


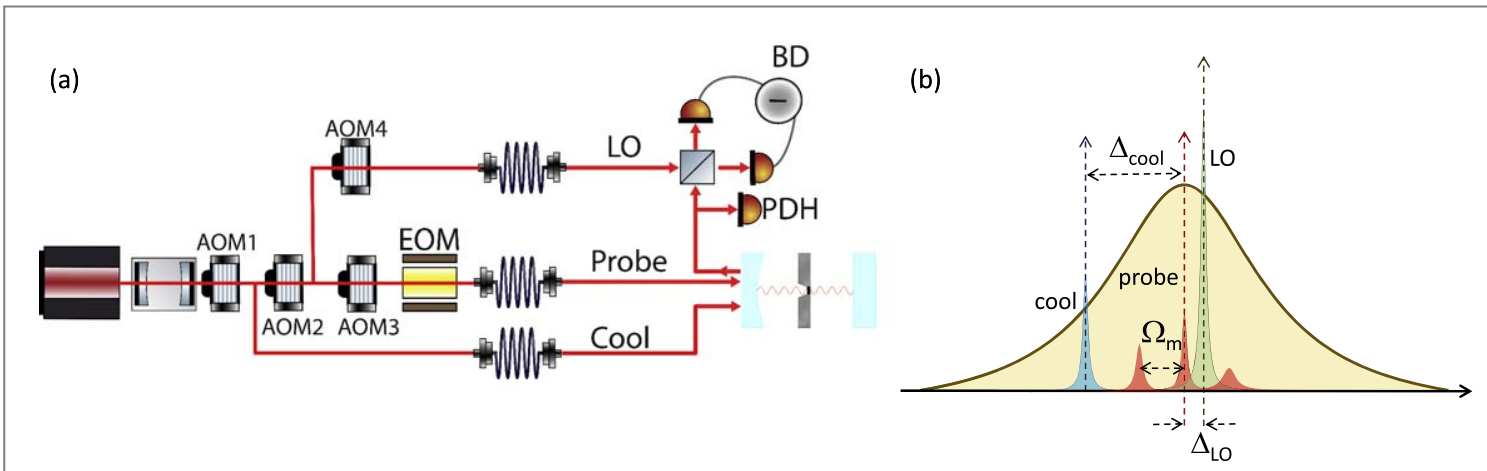

Figure 1. (a) Simplified scheme of the experimental setup. The light of a Nd:YAG laser is filtered by a cavity having a linewidth of $66 \mathrm{kHz}$, frequency tuned by a first acousto-optic modulator (AOM), and split into three parts. The first beam (probe) is frequency shifted by two cascade AOMs acting on opposite orders, and phase modulated by an electro-optic modulator (EOM) at $13 \mathrm{MHz}$ for the Pounder-Drever-Hall (PDH) locking to the resonance of the optomechanical cavity (OMC). The difference between the frequencies of the cascade AOMs defines the detuning of the second beam (cooling beam). The third beam (local oscillator, LO) is picked up after the second AOM, and frequency shifted by a fourth AOM. Its detuning with respect to the probe is defined by the frequency difference between the third and fourth AOMs. After single-mode fibers, the first two beams are combined with orthogonal polarizations and mode-matched to the OMC. About $2 \mu \mathrm{W}$ of the reflected probe are sent to the PDH detection, while most of the probe light (18 $\mu \mathrm{W}$ typically impinge on the cavity) is combined with the LO in a balanced detection (BH). (b) Scheme of the field frequencies. The LO is placed on the blue side of the probe and detuned by $\Delta_{\text {LO }} \ll \Omega_{\mathrm{m}}$, therefore the Stokes lines are on the red side of the LO, while the anti-Stokes lines are on the blue side. In the heterodyne spectra, they are located respectively at $\Omega_{\mathrm{m}}+\Delta_{\mathrm{LO}}$ (Stokes) and $\Omega_{\mathrm{m}}-\Delta_{\mathrm{LO}}$ (anti-Stokes).

nominally given by the expression $J_{m}\left(\alpha_{m n} r\right) \cos m \theta$ and $J_{m}\left(\alpha_{m n} r\right) \sin m \theta$, where $(r, \theta)$ are polar coordinates and $r$ is normalized to the membrane radius.

The oscillator is placed in a Fabry-Perot cavity of length $4.38 \mathrm{~mm}$, at $2 \mathrm{~mm}$ from the cavity flat end mirror, forming a 'membrane-in-the-middle' setup [25]. The input coupler is concave with a radius of $50 \mathrm{~mm}$, originating a waist of $70 \mu \mathrm{m}$. The cavity finesse and linewidth are respectively 24500 and $\kappa=1.4 \mathrm{MHz} \times 2 \pi$. The cavity optical axis is displaced from the center of the membrane by $\sim 0.2 \mathrm{~mm}$, roughly along the axis with $\theta \simeq 0$. As a consequence, the optomechanical coupling and readout are much more efficient for one of the modes in each quasi-degenerate couple (with the shape $\propto \cos m \theta$ ), that we identify as 'light twin', with respect to the other one ('heavy twin').

In this work we mainly focus on the $(1,1)$ modes at $370 \mathrm{kHz}$, having a quality factor of $8.9 \times 10^{6}$ at cryogenic temperature, which leads to an intrinsic width $\Gamma_{m} / 2 \pi=40 \mathrm{mHz}$.

The optomechanical cavity is cooled down to $\sim 7 \mathrm{~K}$ in an helium flux cryostat. The light of a Nd:YAG laser is filtered by a Fabry-Perot cavity and split into three beams, whose frequencies are controlled by means of acousto-optic modulators (AOM) (figure 1(a)). The first beam (probe) is always resonant with the optomechanical cavity, to which it is kept locked using the Pound-Drever-Hall detection and a servo loop. This exploits the first AOM to follow fast fluctuations, and a piezo-electric transducer to compensate for long term drifts of the cavity length. The second beam (cooling beam), orthogonally polarized with respect to the probe, is also sent to the cavity and red detuned by roughly half a linewidth $\left(\Delta_{\text {cool }}=-2 \pi \times 700 \mathrm{kHz} \simeq-\kappa / 2\right)$. The third beam is used as local oscillator (LO) in a balanced detection of the probe beam, reflected by the cavity. In such a detection scheme the LO can either be frequency shifted with respect to the probe (we use $\Delta_{\mathrm{LO}} / 2 \pi \sim 9 \mathrm{kHz}$ ), allowing a low-frequency heterodyne detection [26] (see figure 1(b)), or phase-locked to the probe for an homodyne detection of its phase quadrature. The first scheme (heterodyne) is useful to separate the motional sidebands generated around the optical frequency of the probe field, at frequency shifts corresponding to the mechanical modes frequencies. The spectra acquired with the second scheme (homodyne) are calibrated in terms of cavity frequency fluctuations using a calibration tone in the probe field, and are used to measure the variance of the motion of the different membrane normal modes.

\section{Experimental results}

We will focus on the $(1,1)$ membrane modes around $370 \mathrm{kHz}$, and we will start our analysis from the homodyne spectra of our optomechanical system. The power of the cooling beam is increased by steps up to $\sim 50 \mu \mathrm{W}$. The result, as shown in figure 2(a), is a gradual cooling of the light mode with a characteristic increase of $\Gamma_{\text {eff }}$ and a simultaneous red-shift of the mechanical resonance frequency due to so-called optical spring effect. On the other hand, the 'heavy twin' mode is weakly coupled to the radiation since the optical spot is close to its nodal axis, therefore the associated spectral peak at $370 \mathrm{kHz}$ shows negligible optomechanical effects. 

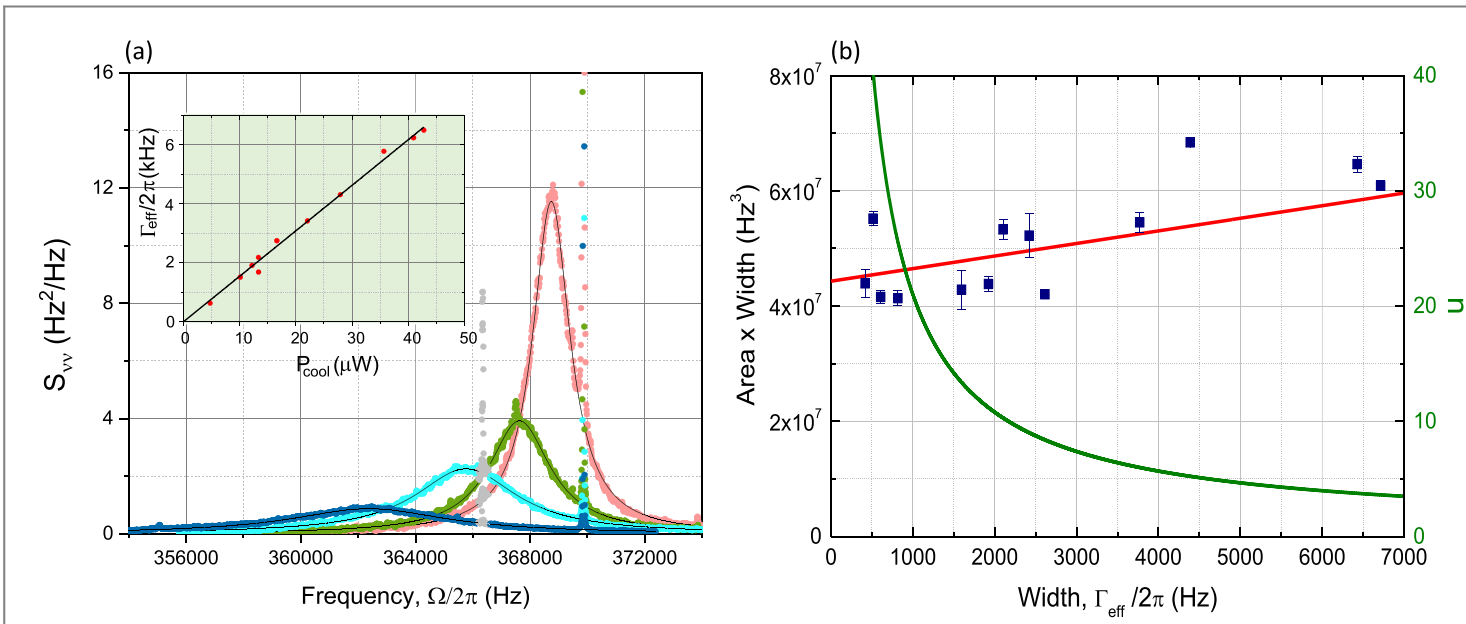

Figure 2. (a) Homodyne spectra around the frequency the $(1,1)$ mechanical modes as the cooling power is increased up to $\sim 50 \mu \mathrm{W}$, maintaining a detuning of $\Delta_{\text {cool }} \sim-\kappa / 2$. The spectra are calibrated in terms of cavity frequency noise $S_{\nu /}$, and are shown without any background subtraction. The peak associated to the 'light twin' mode (strongly coupled to the radiation) exhibits the expected redshift (negative optical spring) and optical cold damping (increase in its width $\Gamma_{\text {eff }}$ and decrease in the peak area). At $\sim 370 \mathrm{kHz}$ is visible the narrow peak due to the 'heavy twin' mode. Symbols show the experimental data, solid lines are the Lorentzian functions fitting the peaks of the 'light twin' modes. A spurious electronic peak is shown with light grey symbols. The inset shows the measured peak width $\Gamma_{\text {eff }} / 2 \pi$ as a function of the cooling power, together with a linear fit. (b) Increment of the measured area $\times$ width product for the strongly coupled $(1,1)$ mode, as a function of its width $\Gamma_{\text {eff }} / 2 \pi$. The red straight line reports the prediction of equation (4), where just an overall scaling factor is fitted to the data. A solid green line shows the mean occupation number $\bar{n}$ calculated according to equation (3).

The decrease of the peak area is an indication of the reduction of the phonon occupancy $\bar{n}$ in the 'light twin' mode. A quantitative evaluation of $\bar{n}$ from this parameter would require an independent measurement of the optomechanical coupling strength. On the other hand, the cooling factor $\Gamma_{\text {eff }} / \Gamma_{\mathrm{m}}$ can here be accurately measured, but it provides a good estimate of the oscillator effective temperature and consequently of its occupation number $\bar{n} \approx \bar{n}_{\mathrm{th}} \Gamma_{\mathrm{m}} / \Gamma_{\text {eff }}$ just in the classical limit (as soon as the back-action is negligible) and in the absence of extra noise. The two indicators can be usefully put together in the area $\times$ width product, that is shown in figure 2(b) as a function of $\Gamma_{\text {eff }}$. The reported values of $\mathcal{A} \Gamma_{\text {eff }}$ are obtained from fits of the spectral peaks with a Lorentzian function. Equation (4) predicts that the $\mathcal{A} \Gamma_{\text {eff }}$ versus $\Gamma_{\text {eff }}$ data should display a linearly increasing behavior, where the slope-to-offset ratio is determined by the different contributions to $\bar{n}$. We have calculated such contributions using independent measurements, as follows. $\bar{n}_{\text {th }}$ is calculated from the bath temperature measured by a silicon diode sensor fixed on the cavity (with an accuracy of $0.1 \mathrm{~K}$ ), and the oscillator frequency. $\bar{n}_{\mathrm{BA}}^{\text {cool }}$ is calculated from equation (1) using the measured cavity linewidth and the detuning $\Delta_{\text {cool }}$ fixed with the AOMs. $\bar{n}_{\mathrm{BA}}^{\text {probe }}$ is calculated from equation (2) assuming $\Delta_{\text {probe }} \simeq 0$, measuring the probe-to-cooling beam power ratio and fitting the linear dependence between $P^{\mathrm{cool}}$ and $\Gamma_{\mathrm{eff}}$ (see the inset in figure 2(a)). Finally, $\Gamma_{\mathrm{m}}$ is obtained from ring-down measurements with an additional laser at $970 \mathrm{~nm}$, where the measured optomechanical effects are very weak due to the low cavity finesse and laser power. The experimental measurements well follow the predicted slope, shown with a solid line in figure 2(b), where just the overall vertical scaling factor is fitted to the data. Here the error bars just reflects the standard deviation of measurements performed on consecutive acquisitions. The scattering of the data shows that longer term fluctuations in system parameters (when changing the cooling power) dominate over such statistical uncertainties, that are therefore not considered as meaningful in the following analysis.

A further solid curve in the figure shows the behavior of $\bar{n}$ calculated from equation (3), i.e. assuming that the system is well modeled and in the absence of additional noise, as suggested by the good agreement between the prediction of equation (4) and the experimental data. We infer that an occupation number of $\bar{n}=3.9$ is achieved at our maximum cooling power. Moreover, the fitted vertical scaling factor allows to estimate, according to equation (4), a vacuum optomechanical coupling strength of $g_{0} / 2 \pi=31 \pm 1 \mathrm{~Hz}$. We remark again that such additional inferred parameter is not involved in the evaluation of $\bar{n}$.

The analysis so far assumes that the model well describes the system behavior. However, the observed qualitative agreement is not yet a safe guarantee of an accurate measurement. Heating of the membrane oscillator due to laser absorption would yield a linear increase of $T_{\text {bath }}$ with $P_{\text {cool }}$, and thus a larger slope of $\mathcal{A} \Gamma_{\text {eff }}$ versus $\Gamma_{\text {eff }}$. Leaving the slope as free parameter in the fit of $\mathcal{A} \Gamma_{\text {eff }}$ versus $\Gamma_{\text {eff }}$, we find for the ratio between slope and offset a value of $(8.0 \pm 2.5) \times 10^{-5} \mathrm{~Hz}^{-1}$, to be compared with $4.9 \times 10^{-5} \mathrm{~Hz}^{-1}$ calculated from equation (4). This suggests that the sample temperature could have increased by $1.8 \pm 1.5 \mathrm{~K}$ at our maximum cooling power, for an achieved occupation number of $\bar{n}=4.0 \pm 0.5$. 

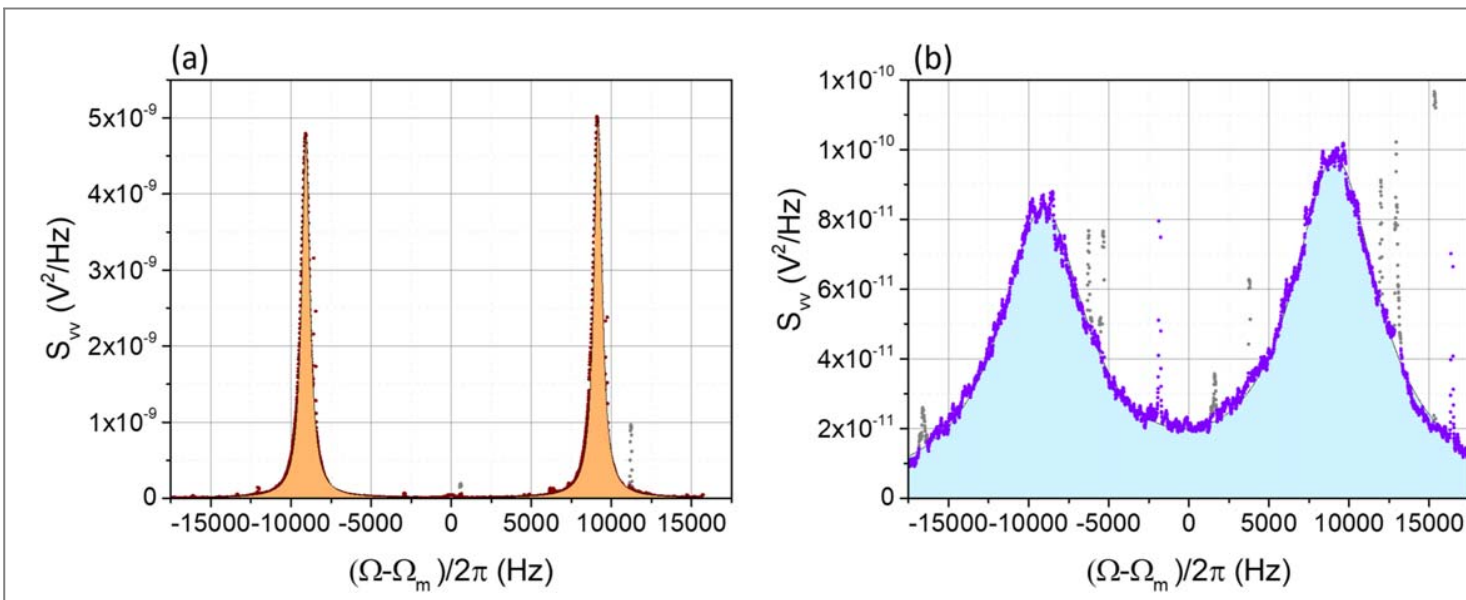

Figure 3. Observation of the Stokes (right) and anti-Stokes (left) spectral peaks of the $(1,1)$ membrane mode for two different values of the cooling power: (a) at low cooling power the spectral width $\Gamma_{\text {eff }}$ is still relatively small and the sideband asymmetry is just $R \simeq 1.06$, yielding an inferred mean phonon occupancy of $17.1 \pm 3.4$. (b) At larger cooling power, producing broader peaks, the asymmetry is more evident, with a measured value of $R \simeq 1.24$ and a mean phonon occupancy of $3.87 \pm 0.21$. Symbols show the experimental data, including the narrow peaks of the 'heavy twin' mode and spurious electronic peaks shown in light grey. Solid lines are the fitting functions, composed of couples of Lorentzian peaks of equal width and shifted by $2 \Delta_{\mathrm{LO}} / 2 \pi$, plus a linearly decreasing background that is subtracted from the displayed data for the sake of clarity. The fitted mean resonance frequency is taken as origin of the displayed horizontal axis.

Furthermore, additional amplitude or frequency noise in the laser radiation would instead provide a quadratic term in $\mathcal{A} \Gamma_{\text {eff }}$ versus $\Gamma_{\text {eff }}$. We have indeed added such term to the fit of our data, finding a maximum contribution of $13 \% \pm 10 \%$ to the measured $\mathcal{A}$. In both fitting procedures the uncertainty is due to the scattering of the experimental data, and the results are compatible with null effects of heating and extra laser noise. We have measured the intensity and frequency noise of our laser source before entering the optomechanical cavity (after filter cavity, modulators and fibers) obtaining $1+P / 0.5 \mathrm{~mW}$ for the intensity (normalized to shot noise, where $P$ is the laser power) and an upper limit of $0.05 \mathrm{~Hz}^{2} \mathrm{~Hz}^{-1}$ for the frequency fluctuations (measured with respect to an empty reference cavity). For the former we calculate a maximum contribution of less than $2 \%$ to the peak area. The latter could instead justify an increase of up to $15 \%$ in the peak area, corresponding to a raise of 0.7 in $\bar{n}$, at the maximum cooling power.

While the described analysis of the homodyne spectra at increasing cooling power gives an estimate of $\bar{n}$, skipping the independent calibration of the optomechanical coupling, we see that uncertainties in additional noise sources can reduce the measurement accuracy. Therefore, the measurement of the motional sidebands ratio in heterodyne spectra remains in our opinion a superior procedure. Indeed, it gives directly access to the real average phonon occupation number for each value of cooling power, including implicitly extra heating and noise and without the necessity of further independent measurements of system parameters.

Our setup can easily switch from homodyne to heterodyne detection, by just including a frequency offset $\Delta_{\text {LO }}$ in the phase locking of the LO. Figure 3 shows two examples of heterodyne spectra, again around the resonance frequency of the $(1,1)$ modes, for two different values of the cooling power. At low power (panel a) the motional sidebands are very similar, while at higher cooling power (panel b) the increased width $\Gamma_{\text {eff }}$, indicating a smaller occupation number, is accompanied by a visible asymmetry, with a smaller left (anti-Stokes) sideband. For a correct evaluation of $\bar{n}$ one must consider the filtering effect of the cavity, and in particular evaluate the residual probe detuning. To this purpose, we have exploited the motional sidebands of the 'heavy twin' mode that, being weakly coupled to the optical radiation, maintains a occupation number so high that a possible sideband asymmetry should be completely attributed to the cavity filtering effect. Operatively, we measure the sidebands ratio $R_{\text {light }}$ for the 'light twin' mode and $R_{\text {heavy }}$ for the 'heavy twin' mode, and correct the former according to $R=R_{\text {light }} / R_{\text {heavy. }}$. We use this corrected $R$ to estimate $\bar{n}$, that assumes, e.g. the value of $\bar{n}=17.1 \pm 3.4$ for the spectra in panel (a) and $\bar{n}=3.87 \pm 0.21$ for panel (b) (the reported uncertainty is the standard deviation in 10 measurements, performed on consecutive, $10 \mathrm{~s}$ long time intervals, for a total measurement time of $100 \mathrm{~s}$ ). The latter value is obtained for our maximum cooling rate.

This described calibration procedure relies on the close proximity of the resonance frequencies of the two (1, 1) modes, yielding the same cavity filtering effect. However, one can also evaluate the sideband ratio for several weakly coupled modes, deduce the probe detuning $\Delta_{\text {probe }}$ by fitting the results with the function $\mathcal{L}\left(\Delta_{\text {probe }}-\Omega_{\mathrm{m}}\right) / \mathcal{L}\left(\Delta_{\text {probe }}+\Omega_{\mathrm{m}}\right)$ versus $\Omega_{\mathrm{m}}$, and finally use the same function with the inferred $\Delta_{\text {probe }}$ and $\Omega_{\mathrm{m}}=2 \pi \times 370 \mathrm{kHz}$ to correct $R_{\text {light }}$. An example of such fit is shown in figure 4 . This procedure also allows to monitor the stability of the detuning during the measurement, as shown in the inset of figure 4 . We have found 


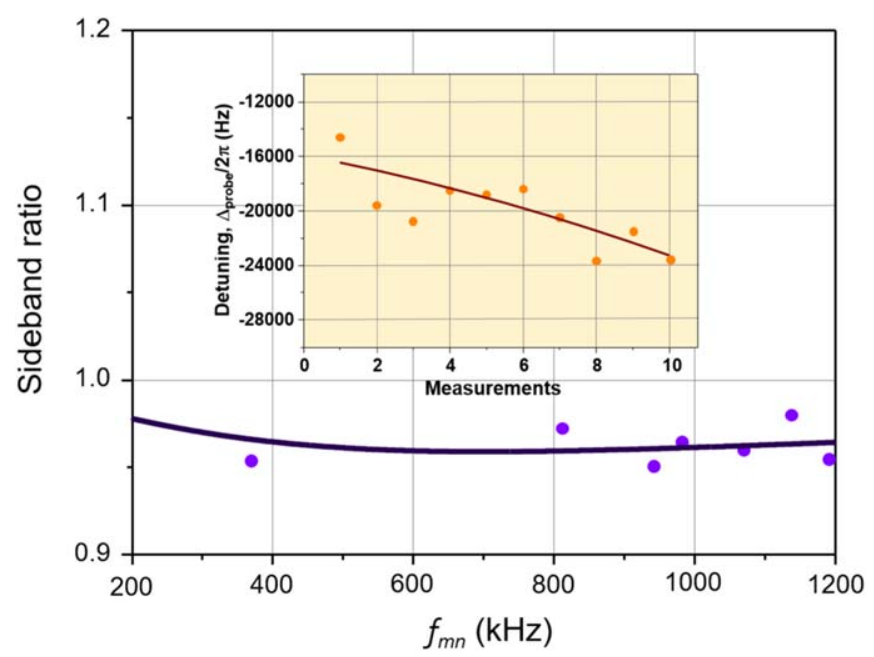

Figure 4. Method for correcting the sideband asymmetry due to the residual probe detuning. The measured sideband ratio for several weakly coupled modes is plotted as a function of the respective resonance frequencies $\Omega_{\mathrm{m}}$ (blue dots), and fitted with the function $\mathcal{L}\left(\Delta_{\text {probe }}-\Omega_{\mathrm{m}}\right) / \mathcal{L}\left(\Delta_{\text {probe }}+\Omega_{\mathrm{m}}\right)$ to infer the probe detuning $\Delta_{\text {probe }}$ (solid line). This procedure is repeated for several consecutive, 10 s long time intervals. The evolution of the inferred values of the detuning (shown with orange close circles in the inset) is fitted with a first or second order polynomial function (solid line in the inset).

typically a detuning below $\mid \Delta_{\text {probel }} / 2 \pi<30 \mathrm{kHz}$ (corresponding to $0.02 \kappa$ ) and variations during a complete measurement three times smaller. The consequent corrections to $R_{\text {light }}$ arrive to nearly $10 \%$. A preliminary evaluation of the sideband ratio for the weakly coupled modes is indeed a method to adjust the probe detuning at the beginning of the measurement. The corrections to $R_{\text {light }}$ obtained with this procedure are in good agreement with the method using directly the 'heavy twin' mode.

The occupation number $\bar{n}$ calculated from the corrected sideband ratio is shown in figure 5(b) as a function of $\Gamma_{\text {eff }}$, obtained at increasing values of cooling power. Filled solid curves reflect the expected $\bar{n}$ and its different contributions, calculated according to equations (1)-(3). In particular, $n_{\mathrm{BA}}^{\text {cool }} \sim 0.58$, showing that for the $(1,1)$ modes we are here in weakly resolved sidebands regime and back-action cooling can in principle bring these modes to an occupation number below unity (close to $n_{\mathrm{BA}}$ in the weak coupling regime [18]). With respect to the analysis of the results extracted from the homodyne spectra, described in figure 2(b), here the theoretical curves have no free fitting parameters: all the contributions to $\bar{n}$ are calculated on the basis of independent measurements. They agree with the experimental data, considering the experimental statistical uncertainty, suggesting the absence of non-modeled extra noise. Each single data point can thus be exploited to extract the occupation number, using as experimental error its statistical uncertainty that, differently from the case of $\mathcal{A} \Gamma_{\text {eff }}$, is now compatible with the data scattering. On the other hand, the overall set of data could be used evaluate $T_{\text {bath }}$, leaving $\bar{n}_{\text {th }}$ as free parameter in the expression (3). With such procedure, the extracted value is $6.7 \pm 0.6 \mathrm{~K}$, compatible with the $7.2 \mathrm{~K}$ measured by the sensor.

\section{Conclusions}

We compare two indicators of the oscillator occupation number, namely the peak area $\times$ width product of the spectrum acquired in a homodyne setup, and the motional sideband asymmetry, measured by heterodyne detection. Neither case requires additional calibrations, even if the actual oscillator base temperature can be an issue for the homodyne method. Moreover, an additional absolute calibration of the homodyne spectrum in terms of frequency fluctuations allows to additionally infer the single-photon optomechanical coupling strength $g_{0}$. Both indicators are particularly sensitive at low occupation numbers (i.e. in the transition from classical to quantum regime). In optomechanical systems where the quantum back-action can be increase until it strongly dominates the thermal noise, the evaluation of $\bar{n}$ from homodyne spectra is facilitated by accessing the region where $\bar{n} \simeq \bar{n}_{\mathrm{BA}}^{\text {cool }}$ [20]. In our case the two kinds of estimate are in agreement, showing that a minimal occupation number of 3.9 is achieved in our experiment. However, we find that the latter indicator is superior because it is less sensitive to additional technical noise, and it gives a result with a single measurement while the former procedure requires a set of measurements as a function of, e.g. the cooling power.

To reliably exploit the latter indicator one should keep in mind that a crucial requirement for an accurate measurement of the sidebands ratio is the control of the probe detuning. We show a method to perform it, based on the observation of the spectral features of weakly coupled mechanical mode. The calibration of the detuning 




Figure 5. Close symbols report the occupation number $\bar{n}$ calculated from the corrected values $R$ of the sideband ratio for the light twin' mode, according to $\bar{n}=1 /(R-1)$. Error bars reflect one standard deviation in the measurements performed on 10 consecutive, 10 s long time intervals. The red solid curve represents the occupation number $\bar{n}$ calculated according to equation (3) using independently measured parameters. Red, green and blue areas represent, respectively, the contributions of the thermal noise, the probe field back-action, and the cooling field back-action.

is thus performed using phase signals generated inside the optomechanical cavity. We find that the use of this method is more trustworthy than exploiting frequency modulation of the probe field since a commonly occurring simultaneous phase and amplitude modulation, as well as spurious reflections along the path of the probe beam, generates asymmetric sidebands that spoils accurate measurements of the cavity detuning.

A widespread use of reliable quantum optomechanical indicators, toward which this work is contributing, is expected to play in important role in the development of quantum technologies [27].

\section{Acknowledgments}

Research performed within the Project QuaSeRT funded by the QuantERA ERA-NET Cofund in Quantum Technologies implemented within the European Unions Horizon 2020 Programme. The research has been partially supported by INFN (HUMOR project). AP has received funding from the European Unions Horizon 2020 research and innovation programme under the Marie Sklodowska-Curie Grant Agreement No. 749709.

\section{ORCID iDs}

A Chowdhury (10 https://orcid.org/0000-0002-1668-8829

A Pontin (iD https://orcid.org/0000-0002-9705-697X

F Marin (10 https://orcid.org/0000-0001-8227-124X

\section{References}

[1] Aspelmeyer M, Kippenberg T J and Marquardt F 2014 Rev. Mod. Phys. 861391

[2] Khalili F Y, Miao H, Yang H, Safavi-Naeini A H, Painter O and Chen Y 2012 Phys. Rev. A 86033840 
[3] Weinstein A J, Lei C U, Wollman E E, Suh J, Metelmann A, Clerk A A and Schwab K C 2014 Phys. Rev. X 4041003

[4] Børkje K 2016 Phys. Rev. A 94043816

[5] Jayich A M, Sankey J C, Børkje K, Lee D, Yang C, Underwood M, Childress L, Petrenko S M, Girvin S M and Harris J G E 2012 New J. Phys. 14115018

[6] Safavi-Naeini A H, Chan J, Hill J T, Gröblacher S, Miao H, Chen Y, Aspelmeyer M and Painter O 2013 New J. Phys. 15035007

[7] Wineland D J, Itano W M, Berquist J C and Hulet R G 1987 Phys. Rev. A 362220

[8] Diedrich F, Berquist J C, Itano W M and Wineland D J 1989 Phys. Rev. Lett. 62403

[9] Purdy T P, Grutter K E, Srinivasan K and Taylor J M 2017 Science 3561265

[10] Sudhir V, Schilling R, Fedorov S A, Schütz H, Wilson D J and Kippenberg T J 2017 Phys. Rev. X 7031055

[11] Safavi-Naeini A H, Chan J, Hill J T, Mayer Alegre T P, Krause A and Painter O 2012 Phys. Rev. Lett. 108033602

[12] Purdy T P, Yu P-L, Kampel N S, Peterson R W, Cicak K, Simmonds R W and Regal C A 2015 Phys. Rev. A 92031802 R

[13] Underwood M, Mason D, Lee D, Xu H, Jiang L, Shkarin A B, Børkje K, Girvin S M and Harris J G E 2015 Phys. Rev. A 92 061801(R)

[14] Peterson R W, Purdy T P, Kampel N S, Andrews R W, Yu P-L, Lehnert K W and Regal C A 2016 Phys. Rev. Lett. 116063601

[15] Sudhir V, Wilson D J, Schilling R, Schütz H, Fedorov S A, Ghadimi A H, Nunnenkamp A and Kippenberg T J 2017 Phys. Rev. X 7 011001

[16] Arcizet O, Cohadon P-F, Briant T, Pinard M and Heidmann A 2006 Nature 44471

[17] Gigan S, Böhm H R, Paternostro M, Blaser F, Langer G, Hertzberg J B, Schwab K C, Bäuerle D, Aspelmeyer M and Zeilinger A 2006 Nature 44467

[18] Marquardt F, Chen J P, Clerk A A and Girvin S M 2007 Phys. Rev. Lett. 99093902

[19] Gorodetksy M L, Schliesser A, Anetsberger G, Deleglise S and Kippenberg T J 2010 Opt. Exp. 1823236

[20] Rossi M, Mason D, Chen J, Tsaturyan Y and Schliesser A 2018 Nature 56353

[21] Borrielli A et al 2014 Microsyst. Technol. 20907

[22] Borrielli A et al 2016 Phys. Rev. B 94 121403(R)

[23] Serra E et al 2016 AIP Adv. 6065004

[24] Serra E, Morana B, Borrielli A, Marin F, Pandraud G, Pontin A, Prod G A, Sarro P M and Bonaldi M 2018 J. Microelectromech. Syst. 271193

[25] Jayich A M, Sankey J C, Zwickl B M, Yang C, Thompson J D, Girvin S M, Clerk A A, Marquardt F and Harris J G E 2008 New J. Phys. 10 095008

[26] Pontin A, Lang J E, Chowdhury A, Vezio P, Marino F, Morana B, Serra E, Marin F and Monteiro T S 2018 Phys. Rev. Lett. 120 020503

[27] Acin A et al 2018 New J. Phys. 20080201 